\title{
The effect of ball mass on the mechanochemical transformation of a single-component organic system: anhydrous caffeine
}

\author{
Adam A. L. Michalchuk ${ }^{1,2,3, *}$ (D), Ivan A. Tumanov ${ }^{1,4}$ (D), and Elena V. Boldyreva ${ }^{1,4, *}$ ([) \\ ${ }^{1}$ Novosibirsk State University, Novosibirsk, Russian Federation \\ ${ }^{2}$ EaStCHEM School of Chemistry, University of Edinburgh, Edinburgh, UK \\ ${ }^{3}$ EPSRC Centre for Continuous Manufacturing and Crystallisation (CMAC), Edinburgh, UK \\ ${ }^{4}$ Institute of Solid State Chemistry and Mechanochemistry SB RAS, Novosibirsk, Russian Federation
}

Received: 28 February 2018

Accepted: 11 April 2018

Published online:

20 April 2018

(C) The Author(s) 2018

\begin{abstract}
ABSTARCT
Mechanochemical methodologies, particularly ball milling, have become commonplace in many laboratories. In the present work, we examine the effects of milling ball mass on the polymorphic conversion of anhydrous caffeine. By investigating a single-phase system, the rate-limiting step of particle-particle contact formation is eliminated. It is found that larger milling balls lead to considerably faster conversion rates. Modelling of the transformation rate suggests that a single, time-independent rate constant is insufficient to describe the transformation. Instead, a convolution of at least two rate-determining processes is required to correctly describe the transformation. This suggests that the early stages of the transformation are governed only by the number of particle-ball collisions. As the reaction proceeds, these collisions less frequently involve reactant, and the rate becomes limited by mass transport, or mixing, even in originally single-phase systems, which become multi-phase as the product is formed. Larger milling balls are less hindered by poorly mixed material. This likely results from a combination of higher impact energies and higher surface areas associated with the larger milling balls. Such insight is important for the selective and targeted design of mechanochemical processes.
\end{abstract}

\section{Introduction}

Mechanochemistry has found many applications in both academic and industrial sciences. This has historically involved use in mineral processing, but is becoming increasingly common in the fine chemicals and pharmaceutical industries [1]. Mechanochemical methods have been used in the synthesis and preparation of a broad range of molecules and materials [2-5]. Many examples are now available where mechanical treatment has been shown to be a

Address correspondence to E-mail: adam.michalchuk@ed.ac.uk; eboldyreva@yahoo.com 
method providing access to novel molecular materials or has been used to enhance the selectivity and the rates of product formation [6-9]. In recent years, attention has largely turned towards the use of mechanical treatment as a tool for synthesis of new organic and coordination compounds, including pharmaceuticals, as well as for control of their polymorphism [10-14].

Despite the widespread interest in the use of mechanochemistry for these purposes, the mechanisms of these reactions are very poorly understood. Offline techniques are known to be affected by 'ageing' of mechanically treated mixtures, and ex situ methods suffer from deficiencies in sampling and disruption of powder structure (tableting). While offline techniques remain vital to mechanochemical study, their use in identifying reaction pathways and kinetics is limited. These issues prompted the rapid development of techniques to probe mechanochemical reactions in situ and in real time. To date, this has been performed using X-ray powder diffraction at synchrotron sources, laboratory-based Raman spectroscopy, or a simultaneous combination of the two [3, 15-19]. In situ, real-time monitoring has proven a great advantage to identifying processes and control parameters in mechanochemistry. In a number of examples, in situ monitoring of multi-component cocrystallisations has revealed previously unknown reaction intermediates, which in some cases could be isolated if treatment were stopped [20]. The use of in situ, real-time techniques has also allowed insights into the macroscopic rates of mechanochemical processes and has revealed a number of complex phenomena, unknown to solution and gas-phase reactions [19].

In the vast majority of cases, mechanical treatment is used to induce transformations between at least two chemically different phases. In these cases, the rate of transformation is greatly limited by the formation of heterogeneous particle-particle contacts [21]. These are required for any reaction to occur. Mixing efficiency therefore becomes one of the most important parameters of the powder sample. It follows that problems can arise in the case of rheological changes, which can have drastic effect on the flowability and mixing of the sample [19, 22]. The observed reaction rates therefore become a convolution of changes in processability with the true kinetics of the transformation. This can lead to difficulties in the identification of kinetic parameters when studying mechanochemical processes.

Despite the issues that surround kinetic analysis of multi-component transformations, many such studies have been performed. In addition to the qualitative monitoring of mechanochemical transformations, in situ technologies have been used to systematically investigate the effects of various process parameters on the rates and products of transformation. To date, this has included such effects as milling frequency, milling ball mass and temperature [17-19, 23]. This style of investigation is critical for the rational design of targeted mechanochemical procedures.

Mechanically induced polymorphism has been documented on a number of occasions, by both neat and liquid-assisted grinding. Ex situ analysis has demonstrated that the type of mechanical treatment $[14,24,25]$, the addition of liquid [14, 26, 27], a polymer [28, 29] and starting polymorph [14] can all play a role in determining the outcome of a polymorphic transformation. This phenomenon is of particular importance to industry, where the solid form of a material is vital to the integrity of its function and the legalities that surround its applications. However, to the best of our knowledge, although the effect of temperature and duration of milling on a single-component transformation has been reported with reference to amorphisation [30-33] and polymorphic transformations [34-38], little work has been done to analyse the effects of other milling parameters on related processes. To the best of our knowledge, there is currently also no work dedicated to the study of single-phase polymorphic transformations under mechanical treatment, probed by real-time and in situ X-ray powder diffraction techniques. While industrially important, such investigation is of great value for fundamental mechanochemistry, where heterogeneous particle contacts are no longer required. The kinetics revealed by such a study therefore reflects the true rate of the mechanically induced conversion.

In this study, we make use of a pharmaceutically active compound, caffeine. Caffeine has been the subject of immense interest to the solid-state research community [28, 39-42] and has served as a fundamental example of the benefits of co-crystallisation on the physico-chemical properties of drug materials [43]. This compound exists in a variety of solid forms, for which the structures of two anhydrous polymorphs and one monohydrate are known, Fig. 1 
Figure 1 Crystal structures of anhydrous caffeine polymorphs, along the crystallographic $c$-axis. Temperatures and nomenclature are taken according to the literature [40]. The $\alpha$-form is highly disordered about each crystallographic site.

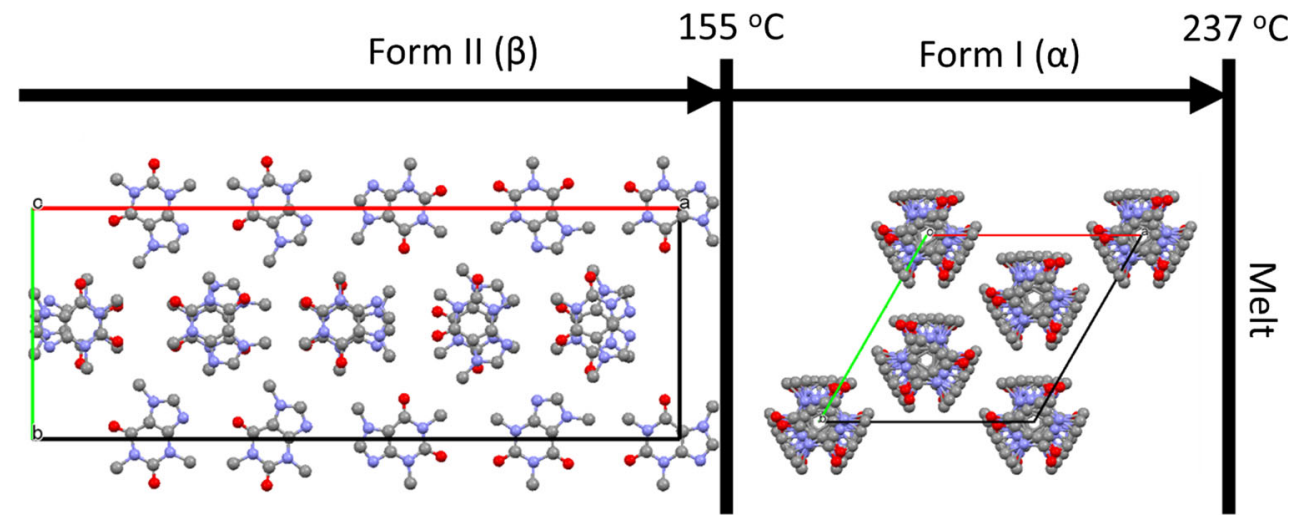

[44, 45]. Both anhydrous polymorphs contain highly disordered caffeine molecules. It has been previously demonstrated that milling of both anhydrous forms leads to a metastable phase [39]. While the structure of this metastable phase remains unknown, the XRPD patterns suggest that the same phase is formed on milling either of the two anhydrous polymorphs. The present study examines the effect of milling parameters on the transformation of the stable form, caffeine II (CAFII), to the metastable intermediate state. In doing so, we present the first example of in situ real-time X-ray powder diffraction applied to a single-phase polymorphic transformation.

\section{Experimental}

\section{Materials}

Anhydrous caffeine (Sigma-Aldrich, 99\%) was used as supplied, without further purification. Polymorph purity was confirmed by X-ray powder diffraction.

\section{Mechanical treatment}

All milling experiments were performed with a MM400 Retsch ball mill, modified for purpose on a synchrotron beam line. For each experiment, $300 \mathrm{mg}$ of pure anhydrous caffeine powder was used. Samples were loaded into Perspex milling vessels (internal volume $14.5 \mathrm{~mL}$ ), which have been previously demonstrated to be suitable for in situ X-ray diffraction experiments $[15,16,19,46]$. Jars were generated in line with the engineering specifications in [47]. A stainless steel ball of $1.4 \mathrm{~g}$ (7 mm diameter; small), $3.4 \mathrm{~g}$ (10 mm diameter; medium) or $13.4 \mathrm{~g}$ (15 mm diameter; large) was added to the vessel, depending on the experiment. Details are provided in the main text. The need for use of only a single ball in studying mechanochemical kinetics is necessary to eliminate stochastic ball-ball collisions [48]. Unless otherwise stated, milling was performed at $30 \mathrm{~Hz}$. All experiments were done without deliberate addition of liquids, though without special drying of the surrounding air.

\section{Diffraction}

All in situ diffraction experiments were performed on the ID-11 beam line at the European Synchrotron Radiation Facility (ESRF). Monochromatic X-rays of wavelength $0.141696 \AA$ were used. Powder patterns were collected every $0.4 \mathrm{~s}$. Data were averaged by summing 10 detector frames, giving a total time resolution of $4 \mathrm{~s}$.

\section{Data processing}

The 2D data were integrated using the PyFAI azimuthal integration method $[49,50]$. Data were subsequently background corrected using the Sonneveld [51] scheme available in the Powder3D software [52]. Patterns were subsequently normalised to a total intensity of 1 to eliminate effects of varying powder in the X-ray [19]. This resulted in no change in the relative intensity of peaks within each pattern. The second derivative curves were plotted.

\section{Results and discussion}

As the structure of the metastable intermediate phase remains unknown, it was not possible to conduct Rietveld refinement of the data obtained from in situ $X$-ray diffraction patterns. For the anhydrous phase of CAFII, the major feature in the diffraction pattern 
is a pair of Bragg peaks at $d$-spacing $=3.30$ and $3.38 \AA$, ESI. Instead, in the intermediate phase these merge into a single, well-defined peak [39]. It is therefore possible to use this transformation to monitor the approximate rate of the transformation. Analysis of the background-corrected XRPD profiles (ESI) does not unambiguously allow detection of the second Bragg peak of the reactant phase. This is particularly true for partially converted samples, where the two reactant peaks are no longer well resolved. Instead, we make use of the derivative properties of curves. That being that the curvature of a peak is proportional to its height and that small 'humps' (e.g. shoulders) can be numerically detected. In this method, we are able to identify any traces of the second reactant peak, including the existence of small shoulder peaks. Provided minimal broadening of the product peak occurs; one therefore expects a complete conversion to result in a plateau at 0 . For all points at which the curve sits below zero, a reactant peak remains.

We do note that this method cannot offer a direct comparison between different samples and only suggests a relative, internal quantification. That said, as the curvature directly reflects the shape of the Bragg peak and thus the crystallinity of the material, powder beginning from the same stock sample should have approximately the same initial value. We also note that small fluctuations can be expected due to statistical broadening from powder position within the vessel.

\section{Mechanical treatment of caffeine form II- $30 \mathrm{~Hz}$}

Samples of CAFII were subjected to ball milling under three experimental conditions. In the first, the sample was milled at $30 \mathrm{~Hz}$ with a single small ball $(7 \mathrm{~mm}$ diameter, $1.4 \mathrm{~g}$ ), Fig. 2a. The reaction profile follows the expected general structure for a finite system and is consistent with observation of the raw XRPD profiles. The early stages of the transformation are marked by rapid conversion to the metastable intermediate phase. There is a high initial rate of conversion for the first ca $350 \mathrm{~s}$, while the proportion of reactant remains high and each powder-ball impact can be assumed to be capable of a reactive collision. The profile is then seen to slow considerably as the reactant is consumed and many ball-particle collisions instead involve the product phase. A plateau is finally found to appear following ca 45 min of continued milling, Table 1. In contrast to many multi-phase kinetic profiles, we note that the polymorphic conversion does not exhibit an induction period. That is that nucleation of the product phase is not the limiting step. This further suggests that initiation of the polymorphic conversion is not dependent on a build-up of energy or temperature, but instead occurs as a direct result of ball-particle collisions.

Mathematically, any rate that is dependent upon the quantity of a reactant component can be described by a series of exponential equations. Relatively simple exponential functions have been previously derived for multi-component mechanochemical reactions, proving to describe a range of mechanochemical systems [48, 53, 54]. Typically, if only a single process is dominant, a single function can be employed of the form:

$\frac{\mathrm{d} \alpha}{\mathrm{d} t} \propto f(\alpha) \exp (\tau)$

where the conversion quantity, $\alpha$, is dependent on an appropriate kinetic law, $f(\alpha)$, and a ratio of required and available energies, $\tau$. In terms of mechanochemistry, provided sufficient energy is available from an impact, and $\tau$ can be approximated as the time-dependent availability of the perturbing energy (i.e. pulses associated with ball-particle impacts). This differs from solution chemistry where the energy profile of the activating energy remains constant. In this view, the conversion rate is proportional to the rate of the chemical or physical transformation itself, as well as the time-dependent injection of energy that is required to instigate the transformation.

For the milling of CAFII, the rate profile of the transformation cannot be modelled using a simple one-component exponential growth function. A single time constant thus cannot be used to model this transformation. Instead, it is found that a two-phase equation perfectly reproduces the rate profile, Fig. 3a. This equation takes the form,

$\frac{\mathrm{d} \alpha}{\mathrm{d} t} \propto f^{\prime}(\alpha)\left(1-\exp \left(\tau^{\prime}\right)\right)+f^{\prime \prime}(\alpha)\left(1-\exp \left(\tau^{\prime \prime}\right)\right)$

with each exponential function taking nearly equal weighting in the fit (1:0.6).

The need to employ such an equation highlights the qualitatively observed fast and slow regimes. When milling with the small milling ball, the former regime is over tenfold more rapid than the slow 

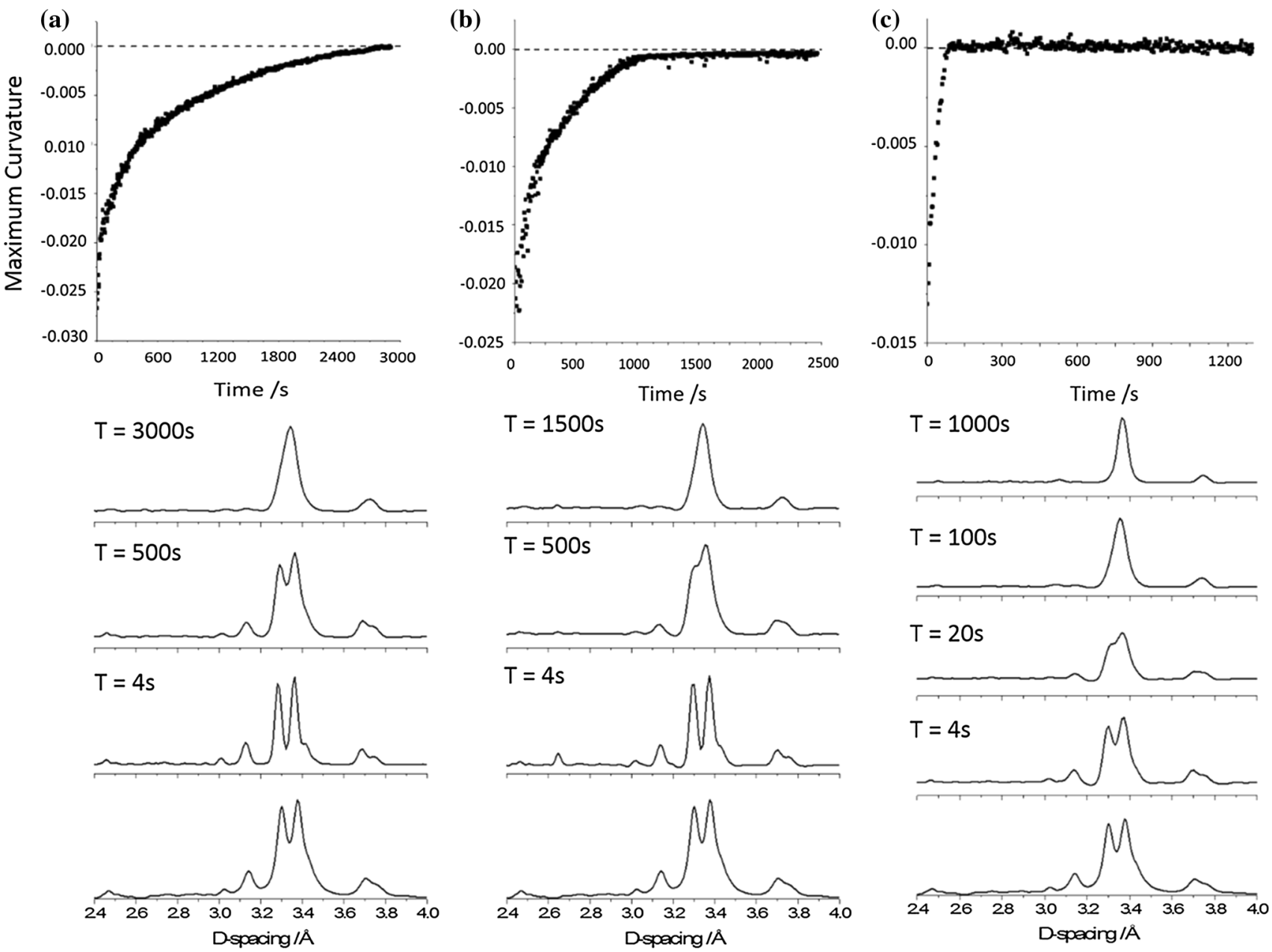

Figure 2 Transformation of CAFII by ball milling at $30 \mathrm{~Hz}$ with a $7 \mathrm{~mm}$ ball, b $10 \mathrm{~mm}$ ball and c $15 \mathrm{~mm}$ ball. The conversion profile is given (top), along with excerpt XRPD patterns at the identified time steps. The bottom XRPD profile is simulated CAFII in each case.

Table 1 Characteristics of milling balls and the resulting times to plateau

\begin{tabular}{llll}
\hline Ball mass $(\mathrm{g})$ & Ball diameter $(\mathrm{mm})$ & Ball surface area $\left(\mathrm{mm}^{2}\right)$ & Approx. plateau time $(\mathrm{s})$ \\
\hline 1.4 & 7 & 153.94 & 2850 \\
3.4 & 10 & 314.16 & 1000 \\
13.4 & 15 & 706.86 & 100 \\
\hline
\end{tabular}

regime. Little physical interpretation can be made from the use of such mathematic constructs in the absence of deeper investigation of an underlying physical model.

However, given current understanding of mechanochemical processes, a plausible mechanism can be discussed. By studying a single-phase system, the need to form reactive contacts is eliminated. Noting that a mechanochemical reaction can occur only on contact with the milling ball, it follows that mass transport remains crucial in the originally single-phase systems, which become multiple-phase as the product phase appears. As the quantity of reactant decreases, the probability that it sits in the direct path of the milling ball at each impact is reduced. This leads to a notable decrease in the conversion rate, limited by mixing.

An increase in the ball mass by approximately twofold ( $10 \mathrm{~mm}$ diameter, $3.4 \mathrm{~g}$ ) leads to a drastic shift in the rate of polymorphic transition, Fig. $2 b$. Again, the same three profile stages are observed. There is a rapid initial conversion, followed by a slowing of the transformation, and finally a plateau is reached. In this case, the plateau is found to arise by 
(a)

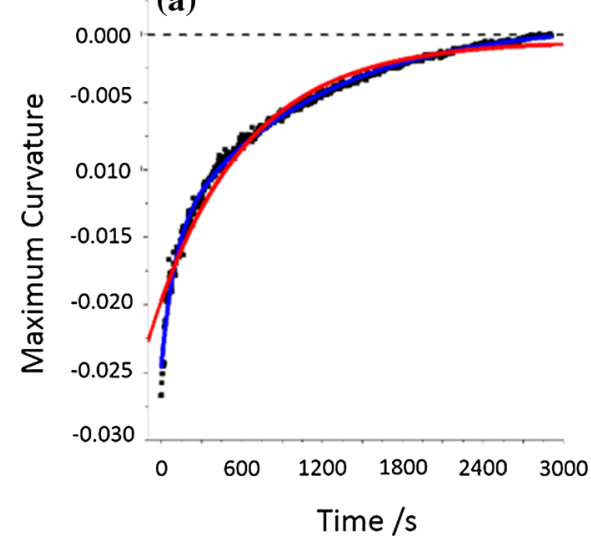

(b)

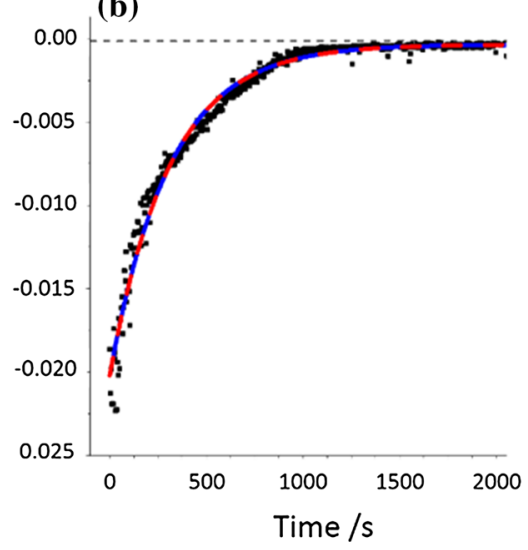

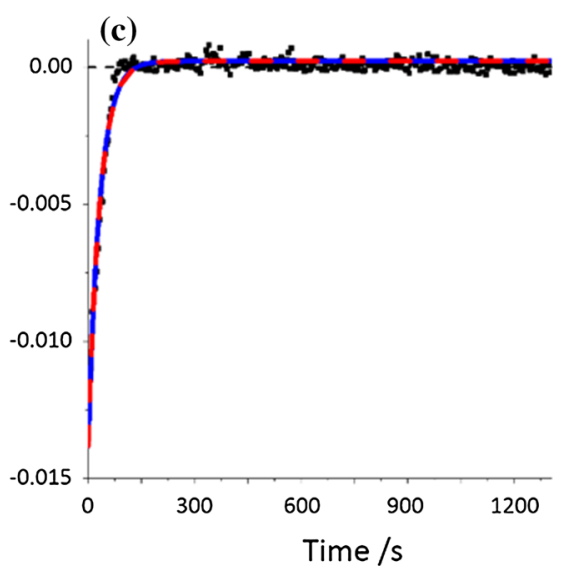

Figure 3 Fitting of multi-phase reaction equations to rate profiles of milling at $30 \mathrm{~Hz}$ with $\mathbf{a}$ small ball, $\mathbf{b}$ medium ball and $\mathbf{c}$ large ball. In each case, a single exponential fit red and double exponential fit blue are given.

approximately $16-17 \mathrm{~min}$ of continued milling, Table 1. This is an approximately threefold increase in the rate of conversion as compared with the previous case. We can again fit the reaction profile to the same multistage equation, Eq. (2), Fig. 3b. In contrast to milling with the smaller ball, the rate constants associated with the 'fast' and 'slow' regimes in this case differ only fivefold. However, the weightings of each exponential become 4:1. In fact, the profile can be fit by employing only the first exponential function, Fig. $3 \mathrm{~b}$. This suggests that the kinetic restriction imposed by mass transport is considerably less with the medium sized ball.

Finally, increasing the ball mass further $(15 \mathrm{~mm}$ diameter, $13.4 \mathrm{~g}$ ) yields a notably larger change than before, Fig. 2c. It is first noted that the initial powder pattern $(T=4 \mathrm{~s})$ already exhibits notable merging of the reactant peaks at d-spacing of 3.30 and $3.38 \AA$, Fig. 3c. This suggests that after only four seconds of milling, notable conversion has already taken place. The conversion profile observed for the transformation with the largest ball appears to have only two stages: a very rapid transformation, followed by the plateau. There is no notable slowing of the conversion rate in this case. The conversion is notably quicker than the previous cases, with the plateau reached in only ca $100 \mathrm{~s}$, Table 1 . This is an approximately tenfold increase in conversion rate, with only fourfold increase in ball mass. Consistent with the previous two examples, we again fit the reaction profile to a multi-phase reaction, Eq. (2). Here, both exponential components adopt exactly the same form and weighting. Thus, the equation reduces exactly to a single function reaction equation. This suggests that there is no limitation imposed by mixing, and Eq. (2) reduces back to a single-component reaction model.

It is clear that the ball characteristics play an important role in driving the rate of this single-phase transformation, and their influence on the transformations is similar to its effect on multi-component reactions. However, the mechanism of this dependence is not clear. In the previous literature, it is generally suggested that the important factor of the milling ball is its mass [17] and that the rate of conversion is proportional to the energy imparted by the associated impact. This may be true for some reactions $[55,56]$. In the present case, ball bass may be associated with more rapid comminution and an increase in the generation of defects per impact. These defect sites and surfaces are known to permit nucleation of polymorphic transitions. However, the lack of induction period suggests that any such nucleation must occur more rapidly than the timescales studied here. An additional factor that one should consider is the area of impact that is associated with each milling ball. That is to note that the number of impacted particles will greatly increase upon increasing the size of the milling ball. This, in turn, reduces any limiting effects of mixing, even in the case of an originally single-phase system. Interestingly, the previous works on the kinetics of mechanochemical reactions have identified a ratelimiting step to be generation of new reactive interfaces, according to an exponential function [54]. When ball-particle interfaces are considered, the rate of contact formation depends on both ball surface area and the rate of comminution of the powder sample. The exact role of the milling ball is certainly complex, 
with the reaction rate depending partially on the energy imposed by each milling ball, the area of impact, the depth of mechanical disturbance on a particle and even the rate of particle size reduction. With current technologies, it is not yet possible to identify which factors are dominant.

\section{Mechanical treatment of caffeine form II- $25 \mathrm{~Hz}$}

As a comparison, a small milling ball was also used to mill CAFII at $25 \mathrm{~Hz}$. As observed at $30 \mathrm{~Hz}$, there is no visible induction period. The transformation begins immediately, albeit with a considerably slower rate than at $30 \mathrm{~Hz}$, Fig. $4 \mathrm{a}$. In the first instance, this is unsurprising as the number of reactive impacts must be correspondingly less when milled at $25 \mathrm{~Hz}$.
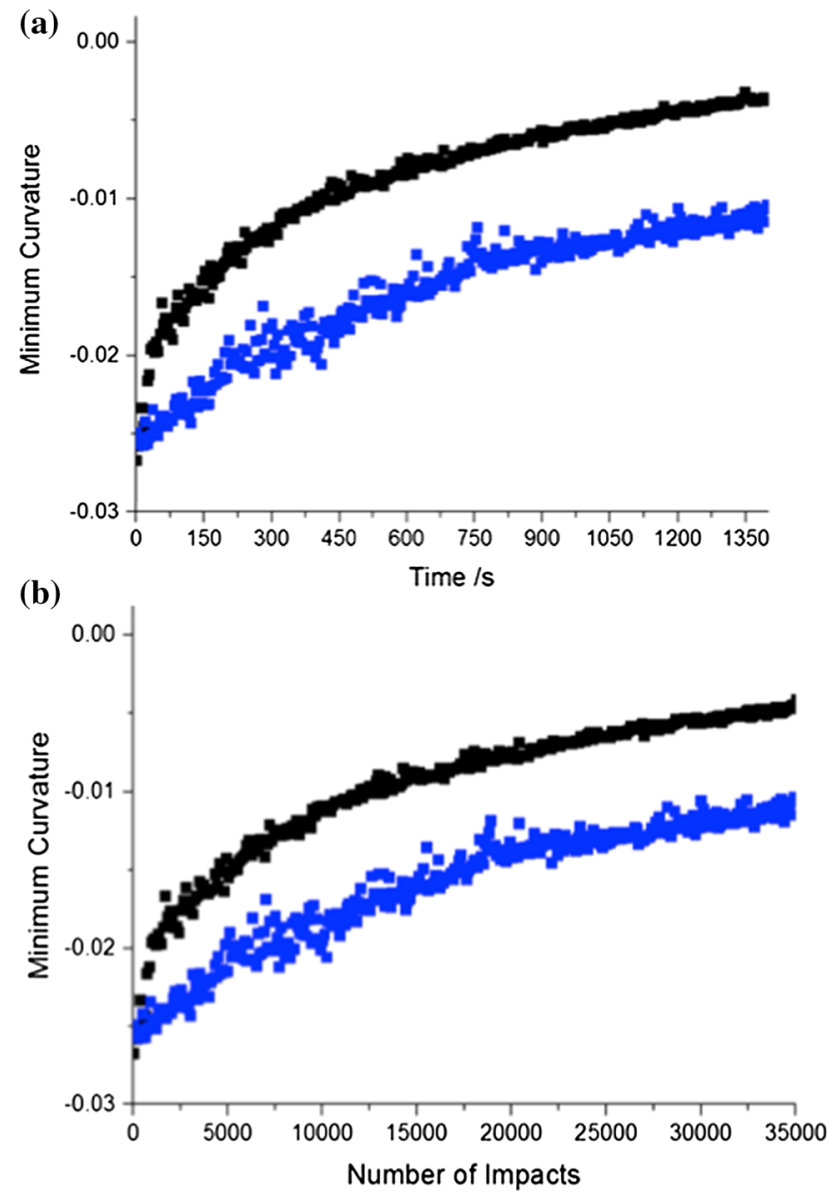

Figure 4 Comparison of conversion rates by milling at $25 \mathrm{~Hz}$ (blue) and $30 \mathrm{~Hz}$ (black). Milling is performed with the smallest milling ball $(1.4 \mathrm{~g})$. a The rate of transformation of CAFII as a function of time and $\mathbf{b}$ normalised for the theoretical number of impacts at each milling frequency.
However, normalisation by the ideal number of impacts continues to display a difference in the rate of conversion, Fig. $4 \mathrm{~b}$. Noting the stochastic motion of a milling ball within a vessel, it is impossible to know the exact number of impacts during any given experiment. That said, it is remarkable that the curves for milling at $25 \mathrm{~Hz}$ and $30 \mathrm{~Hz}$ run parallel except for the initial stages of the transformation. This is particularly apparent when the normalisation by the number of impacts is accounted for. The reason for early divergence of rates is likely due to the energy of the impacts associated with each milling frequency, which directly affects the rate of particle comminution and thus mixing.

\section{Conclusions}

In contrast to solution and gas-phase processes, a mechanochemical reaction occurs only at particleparticle-milling ball interfaces. As such, the rate-determining step in multi-component mechanochemical reactions is formation of reactive interfaces. This hindrance can be removed by studying mechanochemical conversion of single-phase systems. Following the rate of polymorphic transformation of CAFII under milling revealed a sizable dependence between milling ball size and the rate of the transformation. Surprisingly, the rate of transformation cannot be modelled using a time-independent rate equation. This suggests that the dominating rate-determining step changes with time. It can be suggested that in the early stages of the reaction, all collisions occur between the milling ball and reactant CAFII. As the reaction proceeds, however, the probability of reactant-ball collisions decreases, and the rate becomes dominated by mass transport (mixing), since the system becomes twophase as soon as the product is formed. The time dependence of this evolution depends on the milling ball size. It is not yet possible to unambiguously determine the root of this effect. From an energy perspective, larger and heavier milling balls lead to more rapid comminution and increased generation of defects, sites at which polymorphic transitions can nucleate. Larger milling balls also have larger surface areas and as such are less affected by poor mixing. The true effect is likely the result of a combination of these two complex phenomena. Model experiments with balls of variable size and constant mass, or 
alternatively, variable mass and constant size, will shed more light on the problem. Single-phase systems allow drastic reduction in the complexity of the mechanochemical problem under investigation. By employing such systems, new insight can be obtained with regard to the fundamental parameters of mechanochemical processes. This understanding is crucial for the selective and targeted design of mechanochemical reactions.

\section{Acknowledgements}

All experiments described in this study were conducted at the ESRF ID-11 (Experiment CH4313). We acknowledge the assistance of Dr. SAJ Kimber with in situ measurements. The authors thank the Russian 5-100 Programme (AM), EPSRC CMAC EP/I033459/ 1 (AM), an Edinburgh Global Research Scholarship (AM), Grant 16-33-60086 from Russian Foundation of Basic Research (IT) and Project 0301-2018-0007 of Russian Academy of Sciences (EB) for funding. AM thanks Prof. CR Pulham for ongoing support. Final thanks are given to Acad. Prof. VV Boldyrev for many fruitful discussions and advice.

\section{Compliance with ethical standards}

Conflict of interest The authors declare that there are no conflicts to declare.

Electronic supplementary material: The online version of this article (https://doi.org/10.1007/ s10853-018-2324-2) contains supplementary material, which is available to authorized users.

Open Access This article is distributed under the terms of the Creative Commons Attribution 4.0 International License (http://creativecommons.org/ licenses/by/4.0/), which permits unrestricted use, distribution, and reproduction in any medium, provided you give appropriate credit to the original author(s) and the source, provide a link to the Creative Commons license, and indicate if changes were made.

\section{References}

[1] Friscic T, James SL, Boldyreva EV et al (2015) Highlights from Faraday discussion 170: challenges and opportunities of modern. Chem Commun 51:6248-6256

[2] Ali-Moussa H, Navarro Amador R, Martinez J et al (2017) Synthesis and post-synthetic modification of UiO-67 type metal-organic frameworks by mechanochemistry. Mater Lett 197:171-174

[3] Batzdorf L, Zientek N, Rump D et al (2017) Make and break-facile synthesis of cocrystals and comprehensive dissolution studies. J Mol Struct 1133:18-23

[4] Tan D, Friščić T (2018) Mechanochemistry for organic chemists: an update. Eur J Org Chem 2018:18-33

[5] Crawford DE, Casaban J (2016) Recent developments in mechanochemical materials synthesis by extrusion. Adv Mater 28:5747-5754

[6] Beillard A, Bantreil X, Métro T-X et al (2017) Mechanochemistry for facilitated access to N, N-diaryl NHC metal complexes. New J Chem 41:1057-1063

[7] Gonnet L, Tintillier T, Venturini N et al (2017) N-Acyl benzotriazole derivatives for the synthesis of dipeptides and tripeptides and peptide biotinylation by mechanochemistry. ACS Sustain Chem Eng 5:2936-2941

[8] Štrukil V, Igrc MD, Eckert-Maksić M, Friščić T (2012) Click mechanochemistry: quantitative synthesis of "ready to use" chiral organocatalysts by efficient two-fold thiourea coupling to vicinal diamines. Chem Eur J 18:8464-8473

[9] Chow EHH, Strobridge FC, Friscić T (2010) Mechanochemistry of magnesium oxide revisited: facile derivatisation of pharmaceuticals using coordination and supramolecular chemistry. Chem Commun 46:6368-6370

[10] Do JL, Friščić T (2017) Mechanochemistry: a force of synthesis. ACS Cent Sci 3:13-19

[11] Tan D, Loots L, Friščić T (2016) Towards medicinal mechanochemistry: evolution of milling from pharmaceutical solid form screening to the synthesis of active pharmaceutical ingredients (APIs). Chem Commun 52:7760-7781

[12] Fischer F, Heidrich A, Greiser S et al (2016) Polymorphism of mechanochemically synthesized cocrystals: a case study. Cryst Growth Des 16:1701-1707

[13] Štrukil V, Igrc MD, Fábián L et al (2012) A model for a solvent-free synthetic organic research laboratory: clickmechanosynthesis and structural characterization of thioureas without bulk solvents. Green Chem 14:2462-2473

[14] Bouvart N, Palix R-M, Arkhipov S et al (2018) Polymorphism of chlorpropamide on liquid-assisted mechanical treatment: choice of liquid and type of mechanical treatment matter. CrystEngComm 20:1797-1803 
[15] Batzdorf L, Fischer F, Wilke M et al (2015) Direct in situ investigation of milling reactions using combined $\mathrm{x}$-ray diffraction and Raman spectroscopy. Angew Chem Int Ed 54:1799-1802

[16] Ban V, Sadikin Y, Lange M et al (2017) Innovative in situ ball mill for X-ray diffraction. Anal Chem 89:13176-13181

[17] Fischer F, Fendel N, Greiser S et al (2017) Impact is important-systematic investigation of the influence of milling balls in mechanochemical reactions. Org Process Res Dev 21:655-659

[18] Užarević K, Štrukil V, Mottillo C et al (2016) Exploring the effect of temperature on a mechanochemical reaction by in situ synchrotron powder X-ray diffraction. Cryst Growth Des 16:2342-2347

[19] Michalchuk AAL, Tumanov IA, Konar S et al (2017) Challenges of mechanochemistry: Is in situ real-time quantitative phase analysis always reliable? A case study of organic salt formation. Adv Sci 4:1700132

[20] Kulla H, Greiser S, Benemann S et al (2017) Knowing when to stop - trapping metastable polymorphs in mechanochemical reactions. Cryst Growth Des 17:1190-1196

[21] Lapshin OV, Smolyakov VK, Boldyreva EV, Boldyrev VV (2018) Dynamics of the homogenization of binary powder mixtures. Russ J Phys Chem A 92:66-69

[22] Hutchings BP, Crawford DE, Gao L et al (2017) Feedback kinetics in mechanochemistry: the importance of cohesive states. Angew Chemie Int Ed 56:1-6

[23] Kulla H, Wilke M, Fischer F et al (2017) Warming up for mechanosynthesis - temperature development in ball mills during synthesis. Chem Commun 53:1664-1667

[24] Tumanov IA, Achkasov AF, Myz SA et al (2014) Different effect of impact and shear mechanical treatment on mechanochemical cocrystallization of piroxicam and succinic acid. Dokl Chem 457:154-159

[25] Michalchuk AAL, Tumanov IA, Boldyreva EV (2013) Complexities of mechanochemistry: elucidation of processes occurring in mechanical activators via implementation of a simple organic system. CrystEngComm 15:6403

[26] Belenguer AM, Lampronti GI, Cruz-Cabeza AJ et al (2016) Solvation and surface effects on polymorph stabilities at the nanoscale. Chem Sci 7:6617-6627

[27] Cinčić D, Brekalo I, Kaitner B (2012) Solvent-free polymorphism control in a covalent mechanochemical reaction. Cryst Growth Des 12:44-48

[28] Hasa D, Carlino E, Jones W (2016) Polymer-assisted grinding, a versatile method for polymorph control of cocrystallization. Cryst Growth Des 16:1772-1779

[29] Hasa D, Schneider G, Voinovich D, Jones W (2015) Cocrystal formation through mechanochemistry: from neat and liquid-assisted grinding to polymer-assisted grinding. Angew Chemie Int Ed 54:7371-7375

[30] Descamps M, Willart J (2007) Transformation of pharmaceutical compounds upon milling and comilling: the role of Tg. J Pharm Sci 96:1398-1408

[31] Willart JF, Caron V, Descamps M (2007) Transformations of crystalline sugars upon milling. J Therm Anal Calorim 90:125-130

[32] Desprez S, Descamps M (2006) Transformations of glassy indomethacin induced by ball-milling. J Non Cryst Solids 352:4480-4485

[33] Descamps M, Willart JF (2016) Perspectives on the amorphisation/milling relationship in pharmaceutical materials. Adv Drug Deliv Rev 100:51-66

[34] Boldyrev VV (2004) Mechanochemical modification and synthesis of drugs. J Mater Sci 39:5117-5120. https://doi. org/10.1023/B:JMSC.0000039193.69784.1d

[35] Shakhtshneider TP (1997) Phase transformations and stabilization of metastable states of molecular crystals under mechanical activation. Solid State Ionics 101-103:851-856

[36] Shakhtshneider TP, Boldyrev VV (1999) Mechanochemical synthesis and mechanical activation of drugs. In: Boldyreva EV, Boldyrev VV (eds) Reactivity of molecular solids. Wiley, Chichester, pp 271-311

[37] Boldyreva EV (2016) Non-ambient conditions in the investigation and manufacturing of drug forms. Curr Pharm Des 22:1-20

[38] Shakhtshneider TP, Boldyrev VV (1993) Phase transformations in sulfathiazole during mechanical activation. Drug Dev Ind Pharm 19:2055-2067

[39] Hedoux A, Guinet Y, Paccou L et al (2013) Polymorphic transformation of anhydrous caffeine upon grinding and hydrostatic pressurizing analyzed by low-frequency raman spectroscopy. J Pharm Sci 102:162-170

[40] Pinto SS, Diogo HP (2006) Thermochemical study of two anhydrous polymorphs of caffeine. J Chem Thermodyn 38:1515-1522

[41] Madusanka N, Eddleston MD, Arhangelskis M, Jones W (2014) Polymorphs, hydrates and solvates of a co-crystal of caffeine with anthranilic acid. Acta Crystallogr Sect B Struct Sci Cryst Eng Mater 70:72-80

[42] Trask AV, Motherwell WDS, Jones W (2004) Solvent-drop grinding: green polymorph control of cocrystallisation. Chem Comm. https://doi.org/10.1039/b400978a

[43] Trask AV, Motherwell WDS, Jones W (2006) Physical stability enhancement of theophylline via cocrystallization. Int J Pharm 320:114-123

[44] Dichi E, Legendre B, Sghaier M (2014) Physico-chemical characterisation of a new polymorph of caffeine. J Therm Anal Calorim 115:1551-1561 
[45] Enright GD, Terskikh VV, Brouwer DH, Ripmeester JA (2007) The structure of two anhydrous polymorphs of caffeine from single-crystal diffraction and ultrahigh-field solidstate13C NMR spectroscopy. Cryst Growth Des 7:1406-1410

[46] Tumanov N, Ban V, Poulain A, Filinchuk Y (2017) 3Dprinted jars for ball-milling experiments monitored in situ by X-ray powder diffraction. J Appl Crystallogr 50:994-999

[47] Halasz I, Kimber SAJ, Beldon PJ et al (2013) In situ and real-time monitoring of mechanochemical milling reactions using synchrotron X-ray diffraction. Nat Protoc 8:1718-1729

[48] Delogu F, Deidda C, Mulas G et al (2004) A quantitative approach to mechanochemical processes. J Mater Sci 39:5121-5124. https://doi.org/10.1023/B:JMSC.0000039194. 07422.be

[49] Kieffer J, Karkoulis D (2013) PyFAI, a versatile library for azimuthal regrouping. J Phys Conf Ser 425:202012

[50] Kieffer J, Ashiotis G (2014) PyFAI: a Python library for high performance azimuthal integration on GPU. In: 7th European conference on Python in science
[51] Sonneveld EJ, Visser JW (1975) Sonneveld background. J App Chem 8:1-7

[52] Hinrichsen B, Dinnebier RE, Jansen M (2004) Powder3D: an easy to use program for data reduction and graphical presentation of large numbers of powder diffraction patterns. Z Kris 23:231-236

[53] Humphry-Baker SA, Garroni S, Delogu F, Schuh CA (2016) Melt-driven mechanochemical phase transformations in moderately exothermic powder mixtures. Nat Mater 15:1280-1286

[54] Cocco O, Garroni S, Enzo S et al (2018) Ball milling of silica-based pyroclastic scoriae: measurement of mechanochemical reactivity by radical scavenging. J Phys Chem C 122:2773-2782

[55] Fischer F, Wenzel K-J, Rademann K, Emmerling F (2016) Quantitative determination of activation energies in mechanochemical reactions. PCCP 18:23320-23325

[56] Oliveira PFM, Baron M, Chamayou A et al (2016) Lowering the activation energy under mechanochemical conditions: the case of 2,3-diphenylquinoxaline. ChemistrySelect 1:984-988 